\title{
Paramètres de dégradation et de fermentation dans le rumen de rations d'engraissement à base de pulpes séchées complémentées ou non avec du lait ou à base d'ensilage de maïs
}

\author{
AP Mayombo, I Dufrasne, A Clinquart, JL Hornick, L Istasse
}

Faculté médecine vétérinaire, Université de Liège, service de nutrition, Sart Tilman B43, 4000 Liège, Belgique

Trois rations d'engraissement dont 1 à base d'ensilage de maïs (EM) et 2 à base de pulpes séchées (PS) ont été comparées chez 14 taureaux ágés de 15 mois en moyenne et munis d'une canule du rumen. Dans la ration EM (4 taureaux), l'EM (60\% des apports en MS) était complémenté par du tourteau de soja (TS, 10\%), du tourteau de lin (TL, $10 \%)$ et des PS $(20 \%)$. Quatre autres taureaux ont reçu 1 des rations à base de PS composée de $43 \%$ PS, $9 \%$ TS, $9 \%$ TL, $35 \%$ de céréales et $4 \%$ de mélasse. Enfin, un groupe de 6 taureaux a reçu la deuxième ration à base de PS complémentée avec du lait ( $8 \mathrm{~V} / \mathrm{V} /$ animal). Trois taureaux avaient été nourris au seau (PSLS) depuis leur jeune áge jusqu'au début de la période d'engraissement, tandis que les 3 autres avaient été allaités pendant 6 mois (PSLA). Les quantités d'aliments distribuées étaient proches d'ad libitum. La dégradation dans le rumen de 5 aliments (maïs, orge, PS, paille et TL) a été mesurée 4 fois par la technique des sachets de nylon après 4 , $8,16,24$ ou 48 h dincubation. Du liquide du rumen a été prélevé avant le repas du matin et toutes les $2 \mathrm{~h}$ pendant $14 \mathrm{~h}$ afin de déteminer le $\mathrm{pH}$ et de doser les acides gras volatils (AGV) et I'NH $\mathrm{N}_{3}$. Les données ont été traitées par analyse de la variance.

La dégradabilité théorique (DT) de la MS a été supérieure avec la ration EM pour le maïs, les PS et la paille (tableau I). La fraction directement soluble a été systématiquement plus importante avec la ration PSLA, tandis que la fraction non soluble, mais dégradée, a été plus élevée avec la ration PSLS. LeS rations EM ont présenté, par rapport aux rations $P S$, une moindre concentration en AGVT et une proportion plus élevée en acide propionique et en $\mathrm{NH}_{3}$. Le pH a diminué après le repas, davantage avec la ration PSLA $(0,9$ unité $\mathrm{pH})$ qu'avec les autres rations $(0,3,0,4$ et 0,5 unité pH) ; cette chute plus intense étant associée à une concentration supérieure des AGV. La ration PSLA s'est caractérisée également par une proportion plus importante d'acide butyrique et une concentration plus importante de l' $\mathrm{NH}_{3}$.

Les particularités de dégradation des aliments incubés et de digestion enregistrées avec la ration PSLA sont à associer à la fermentation des composants du lait tombés dans le rumen comme le suggère la couleur blanchátre du liquide du rumen prélevé après le repas. À l'opposé, il est très vraisemblable que le réflexe de fermeture de la gouttière cesophagienne ait été maintenu chez les animaux recevant la ration PSLS.

Recherche réalisée avec la collaboration financière de I'IRSIA.

Tableau I. Dégradabilité théorique (DT) et paramètres de fermentation dans le rumen (moyenne des 8 prélèvements journaliers).

\begin{tabular}{lcccc}
\hline Rations & $E M$ & $P S L S$ & $P S L A$ & $P S$ \\
& & & & \\
\hline DT (\%) & & & & \\
Maĩs & $51^{\mathrm{a}}$ & $43^{\mathrm{b}}$ & $44^{\mathrm{b}}$ & $39^{\mathrm{c}}$ \\
Orge & $60^{\mathrm{b}}$ & $54^{\mathrm{c}}$ & $52^{\mathrm{c}}$ & $68^{\mathrm{a}}$ \\
PS & $71^{\mathrm{a}}$ & $57^{\mathrm{b}}$ & $55^{\mathrm{b}}$ & $45^{\mathrm{c}}$ \\
Paille & $21^{\mathrm{a}}$ & $17^{\mathrm{b}}$ & $14^{\mathrm{c}}$ & $20^{\mathrm{a}}$ \\
Tourteau lin & $61^{\mathrm{ab}}$ & $56^{\mathrm{bc}}$ & $64^{\mathrm{a}}$ & $48^{\mathrm{c}}$ \\
PH & $6,6^{\mathrm{a}}$ & $6,5^{\mathrm{ab}}$ & $6,3^{\mathrm{b}}$ & $6,4^{\mathrm{b}}$ \\
AGVT (mmol/) & $11^{\mathrm{c}}$ & $141^{\mathrm{ab}}$ & $154^{\mathrm{a}}$ & $128^{\mathrm{b}}$ \\
$\mathrm{C} 2(\%)$ & $63^{\mathrm{a}}$ & $60^{\mathrm{b}}$ & $56^{\mathrm{b}}$ & $67^{\mathrm{a}}$ \\
$\mathrm{C} 3(\%)$ & $23^{\mathrm{ab}}$ & $21^{\mathrm{b}}$ & $21^{\mathrm{b}}$ & $19^{\mathrm{b}}$ \\
$\mathrm{C} 4(\%)$ & $14^{\mathrm{b}}$ & $17^{\mathrm{b}}$ & $22^{\mathrm{a}}$ & $13^{\mathrm{b}}$ \\
$\mathrm{NH}_{3}(\mathrm{mgN} /)$ & $96^{\mathrm{a}}$ & $66^{\mathrm{b}}$ & $109^{\mathrm{a}}$ & $83^{\mathrm{b}}$ \\
& & & & \\
\hline
\end{tabular}

abc Les moyennes avec la méme lettre dans une ligne $n \Theta$ sont pas significativement différentes $(P<0,05)$. 